\title{
Brownian dynamics: from glassy to trivial
}

H. Jacquin \& F. van Wijland, http://arxiv.org/abs/1206.1586v2

\section{Recommended and with a commentary by Sriram Ramaswamy, IISc, Bangalore}

We all know that altering the dynamical rules governing the evolution of a statistical system, while maintaining detailed balance with respect to the same equilibrium distribution, can alter dramatically the nature and rate of relaxation to thermal equilibrium. Rules constrained by a conservation law lead generically to slower relaxation than nonconserving kinetics [1], and nonlocal or multiparticle moves can speed up relaxation. It is nevertheless surprising when two nearly identical evolution equations, local, number-conserving and relaxing to the same thermal equilibrium distribution, yield drastically different dynamics. One dynamical rule, in standard approximate treatments, displays the mode-coupling glass transition $[2,3]$, while Jacquin and van Wijland (hereafter JvW) show exactly that the other leads to purely exponential relaxation at a rate inversely proportional to the structure factor, with no further enhancement of slowing down by nonlinearities. The same free-energy functional characterizes the equilibrium in both cases, with an abundance of amorphous metastable configurations. JvW's treatment is technical, but their results merit the attention of anyone interested in the reasons behind the slow dynamics of dense, cool, classical simple liquids.

JvW consider a a collection of Brownian particles at temperature $T \equiv 1 / \beta$ interacting through an isotropic pair potential $v(r)$ where $r$ is the interparticle separation. They work in terms of the number density field $\rho=\rho_{0}+\delta \rho(\mathbf{r})$, with mean $\rho_{0}$. The probability of a configuration $\rho$ is $\propto \exp (-\beta F[\rho])$ where

$$
\beta F[\rho]=\int_{\mathbf{r}}\left[\rho \ln \frac{\rho(\mathbf{r})}{\rho_{0}}-\delta \rho(\mathbf{r})\right]+\frac{1}{2} \int_{\mathbf{r}, \mathbf{r}^{\prime}} \delta \rho(\mathbf{r}) v\left(\left|\mathbf{r}-\mathbf{r}^{\prime}\right|\right) \delta \rho\left(\mathbf{r}^{\prime}\right) .
$$

The density functional $F$ and its approximants contain the essential physics of the equilibrium phase transition from liquid to crystal [4]. Minimizing $F$ yields $\rho=\rho_{0} \exp \int_{\mathbf{r}^{\prime}} v(\mid \mathbf{r}-$ $\left.\mathbf{r}^{\prime} \mid\right) \delta \rho\left(\mathbf{r}^{\prime}\right)$, a highly nonlinear integral equation whose solutions include crystalline states for low enough $T$. The abundant occurrence of glassy minima in $F$ is well established [5], especially in a description in which the negative of the direct pair correlation function $c(r)$ 
stands in for $v(r)$. This complex free-energy landscape, a static property, appears to be mirrored consistently in the statistical dynamics [6] of $\rho$ :

$$
\partial_{t} \rho=\nabla \cdot(\rho \nabla \delta F / \delta \rho)+\nabla \cdot(\sqrt{2 T \rho} \mathbf{f})
$$

with a spatiotemporally white, isotropic, unit strength vector noise $\mathbf{f}$ and the Itô interpretation, obeys detailed balance with respect to the Gibbs measure $\exp (-\beta F)$. Eq. (2) shows a glass transition in a one-loop self-consistent treament known as mode-coupling [2, 3]. A similar but seemingly simpler equation,

$$
\partial_{t} \rho=\nabla \cdot\left(\rho_{0} \nabla \delta F / \delta \rho\right)+\nabla \cdot\left(\sqrt{2 T \rho_{0}} \mathbf{f}\right)
$$

in the spirit of dynamical critical phenomena models [1], is obtained by replacing $\rho$ by its mean $\rho_{0}$ at two crucial places in (2), resulting in a constant kinetic coefficient and an additive noise. The strength of nonlinearities in (2) is governed by $v(r)$ and the multiplicative noise, and in (3) by the infinite set of coefficients in an expansion of $\ln \rho / \rho_{0}$. JvW focus their attention on (3), introducing, as in [3], an intermediate field $\theta$ to stand in for the sum of all terms beyond linear order in $\delta \rho$ in the chemical potential $\delta F / \delta \rho$. Using the structure of vertices in the action for the resulting field theory, they are able to show that the selfenergies that would renormalize the dynamics of the two-point space-time correlator of $\rho$ must be strictly zero at all orders in perturbation theory. The relaxation of $\rho$ is determined exactly by an effective linearized (3), with $-v(r)$ replaced by $c(r)$, with a relaxation time $\sim S_{k} / k^{2}$ where $S_{k}$ is the equilibrium static structure factor of $\rho$. On the other hand, we know from work on the mode-coupling glass transition that model (2), with nearly the same kinetics and exactly the same equilibrium distribution, has a far more complex relaxation behaviour.

The findings of $\mathrm{JvW}$ leave us with a lesson, a technical issue, and a conceptual question. The lesson: the mere existence of an exceedingly complex free-energy landscape need not produce complex relaxation. The work underlines the value of deriving continuum stochastic "hydrodynamic" theories by explicitly coarse-graining a microscopic description rather than relying exclusively on considerations of symmetry and conservation, which in this case would very likely miss the subtlety associated with the multiplicative noise. The technical issue: the authors introduce an intermediate field $\theta$ obeying a nonpolynomial constraint, and then proceed to obtain results at all orders in perturbation theory. How is this related to the 
problem in its original formulation without the auxiliary variable? The conceptual question: why does (3) manage to relax so easily, unlike (2)? One possibility: the multiplicative noise in (2) is ultimately [7] a result of the strictly non-negative nature of the underlying field $\rho$, a sum of positive-weight Dirac delta-functions. Does the seemingly harmless modification leading to (3) therefore allow an escape-route in which $\rho$ sometimes gets negative?

[1] P.C. Hohenberg and B.I. Halperin, Rev. Mod. Phys. 49, 435 (1977).

[2] S.P. Das, Rev. Mod. Phys. 76, 785 (2004); D.R. Reichman and P. Charbonneau, J. Stat. Mech. P05013 (2005).

[3] B. Kim and K. Kawasaki, J. Stat. Mech. P02004 (2008)

[4] T.V. Ramakrishnan and M. Yussouff, Phys. Rev. B 19, 2775 (1979).

[5] Y. Singh et al., Phys. Rev. Lett. 54, 1059 (1985); C. Dasgupta, Europhys. Lett. 20, 131 (1992).

[6] D.S. Dean, J. Phys. A: Math. Gen. 29 L619 (1996); for Eq. (2) without noise see B. Bagchi, Physica A 145, 273 (1987).

[7] A. Velenich et al., J. Phys. A: Math. Theor. 41, 235002 (2008); arXiv:0802.3212. 